\title{
Transmission Electron Microscopy of Metal Nanodendrites Grown on Insulator Substrates by Electron-Beam-Induced Deposition
}

\author{
Guoqiang Xie, Minghui Song, Kazutaka Mitsuishi, and Kazuo Furuya
}

High Voltage Electron Microscopy Station, National Institute for Materials Science, Tsukuba 305-0003, Japan

Electron-beam-induced deposition (EBID) is one of the most promising techniques to fabricate small-sized structures on substrates. A variety of nanometer-sized structures have been fabricated [1-3]. Due to stable fabrication, electric conductive substrates are generally used [3] and compact structures are usually fabricated. On the other hand, little attention has been paid for the nanofabrication with insulator substrates. In the present study, metal $(\mathrm{W}, \mathrm{Pt})$ nanodendrites were fabricated on insulator substrates using an EBID process in transmission electron microscopes (TEMs). The as-fabricated microstructure was investigated in detail using conventional and highresolution TEM (CTEM and HRTEM) and X-ray energy dispersive spectroscopy (EDS).

For the fabrication of W-nanodendrites, EBID was carried out in a JEM-ARM 1000 TEM. Accelerating voltages were $400,600,800$, and $1000 \mathrm{kV}$. A gas introduction system including a nozzle and a reservoir of $\mathrm{W}(\mathrm{CO})_{6}$ precursor was installed on this microscope. The nozzle was located near specimen within $2 \mathrm{~mm}$. The inner diameter of the nozzle was smaller than $100 \mu \mathrm{m}$. Crystalline $\mathrm{SiO}_{2}$ TEM thin films were used as substrate. The fabrication of Pt-nanodendrites were carried out on crystalline $\mathrm{Al}_{2} \mathrm{O}_{3}$ substrates. $\mathrm{Me}_{3} \mathrm{MeCpPt}$ powder was a precursor. EBID experiments were done with a JEM-2010F TEM operated at $200 \mathrm{kV}$. All the experiments were carried out at room temperature.

Fig. 1 shows images of $\mathrm{W}$-nanodendrites grown on $\mathrm{SiO}_{2}$ substrate at $400 \mathrm{kV}$. The electron beam is defocused in size of about $2000 \mathrm{~nm}$. The dendrites show a tendency to grow at the edge of the substrate in the irradiated area (Fig. 1a). They are grown self-standing at positions separated from each other in distance of several nanometers. Branches are observed at tips, which thickness is less than $3 \mathrm{~nm}$ (Fig. 1b). The diameter become thicker near the substrate, which imples that the deposition takes place at both tip and trunk part. This growth and morphology are attributed to a mechanism involving electric charge-up produced and accumulated on the surface of the substrate and tips of the deposits [4]. It is confirmed by EDS that $\mathrm{W}$ has been effectively deposited. Further characterization for the as-fabricated dendrites with HRTEM reveals that nano-sized $\mathrm{W}$ grains in bcc structure are contained in the dendrites. Fig. 2 shows HRTEM images of some branches of $\mathrm{W}$-dendrites fabricated with various accelerating voltages. Lattice fringes, which are observed at the most places, indicates the formation of nano-sized crystals. The typical lattice spacing measured from images is $0.22 \mathrm{~nm}$ which is close to the lattice spacing, $0.224 \mathrm{~nm}$, of $\{110\}$ of bcc W crystals. Moreover, the inter-fringe angles of 60 degrees (grain A) and 90 degrees (grain B) agree well with the zone axis of [111] and [001] of bcc W structure, respectively. Furthermore, some lattice fringes shown by arrows in Fig. 2a are not clear because of the co-existence of amorphous state. Fig. 2b shows an image of W-dendrites grown at 1000 $\mathrm{kV}$. Lattice fringes are clearly observed in almost all of the grains. The results indicated that the crystallinity of the dendrites was improved as increase in accelerating voltage. High-energy electron irradiation may enhance the diffusion of $\mathrm{W}$ atoms in the dendrites and promotes the crystallization of $\mathrm{W}$ grains.

With the EBID process, Pt depositions were also carried out on an $\mathrm{Al}_{2} \mathrm{O}_{3}$ substrate. Pt dendrites were observed to grow in the irradiated area, such as the case of $\mathrm{W}$ deposition. The dendrites 
consist of many nano-grains and amorphous state. Fig. 3 is an HRTEM image of tip-part of Ptdendrite. The size of nano-grains is about $3 \mathrm{~nm}$. Lattice fringes are observed clearly. Combined with diffraction pattern and EDS analyses, it is determined to be Pt crystal in fcc structure.

\section{References}

[1] K. Mitsuishi, M. Shimojo, M. Han and K. Furuya: Appl. Phys. Lett. 83 (2003) 2064.

[2] L.X. Dong, F. Arai and T. Fukuda: Appl. Phys. Lett. 81 (2002) 1919.

[3] H.W.P. Koops et al.: Jpn. J. Appl. Phys. Part 133 (1994) 7099.

[4] M. Song, K. Mitsuishi, M. Takeguchi and K. Furuya, Appl. Surf. Sci. 241 (2005) 107.

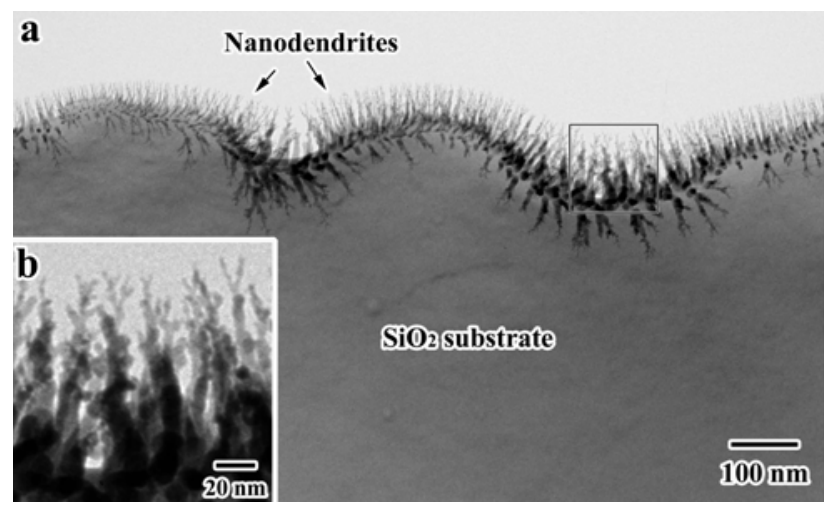

Fig. 1 (a) TEM image of $\mathrm{W}$-nanodendrites grown on $\mathrm{SiO}_{2}$ substrate at $400 \mathrm{kV}$ to a fluence of $6.0 \times$ $10^{21} \mathrm{e} \mathrm{cm}^{-2}$. (b) Enlargement of the square area in (a), showing the nanodendrites in more detail.

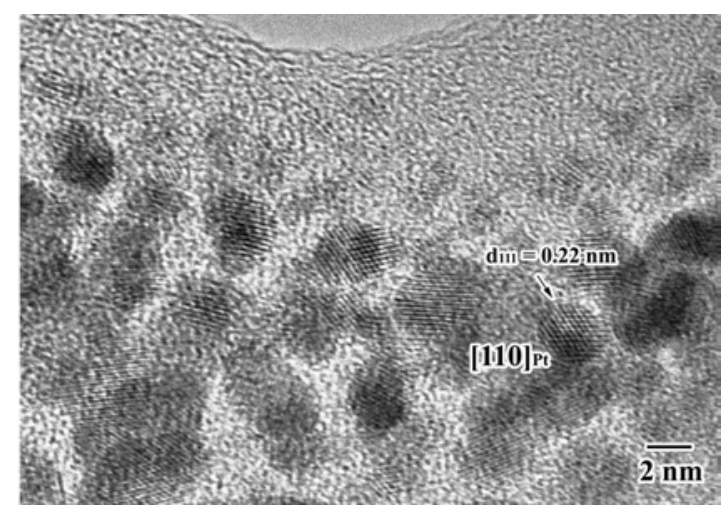

Fig. 3 HRTEM image of tip part of Ptnanodendrite fabricated at $200 \mathrm{kV}$ to a fluence of $2.6 \times 10^{21} \mathrm{e} \mathrm{cm}^{-2}$.
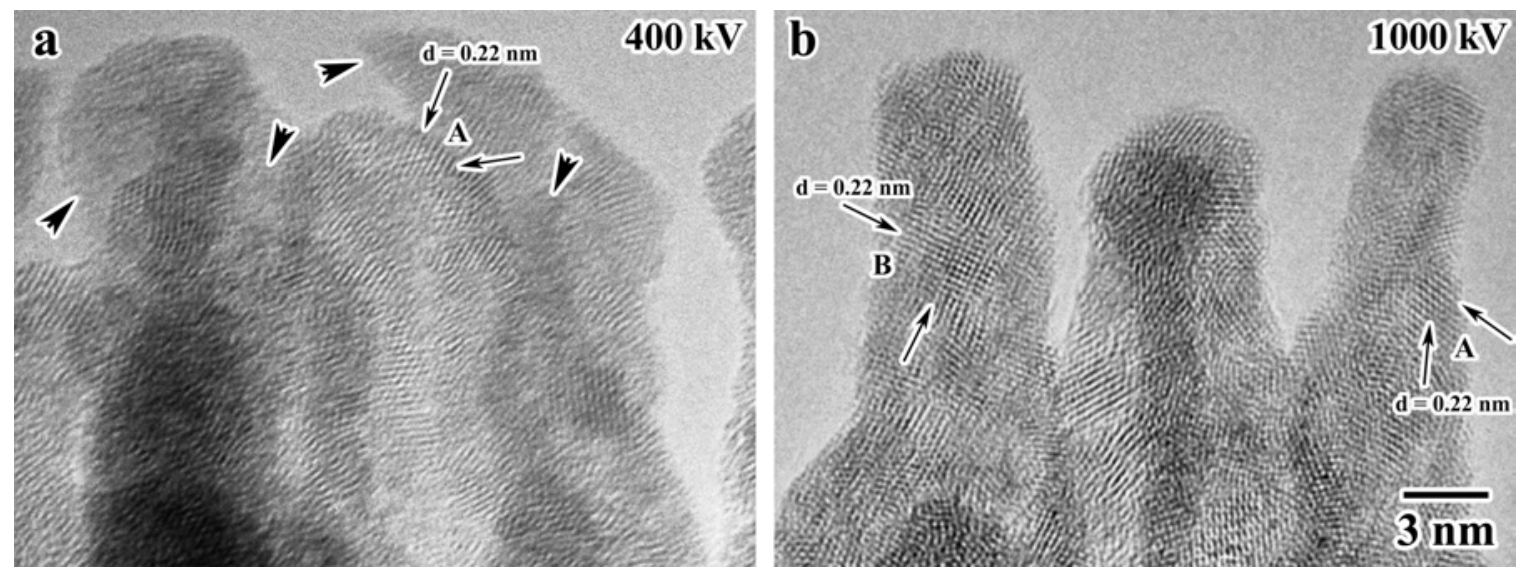

Fig. 2 HRTEM images of some branches of $\mathrm{W}$-nanodendrites grown on $\mathrm{SiO}_{2}$ substrate to a fluence of $6.0 \times 10^{21} \mathrm{e} \mathrm{cm}^{-2}$ with various accelerating voltages. (a) $400 \mathrm{kV}$; (b) $1000 \mathrm{kV}$. Arrows indicate lattice fringes in grains $\mathrm{A}$ and $\mathrm{B}$, which have inter-fringe angles of 60 (A) and 90 (B) degrees, respectively. 